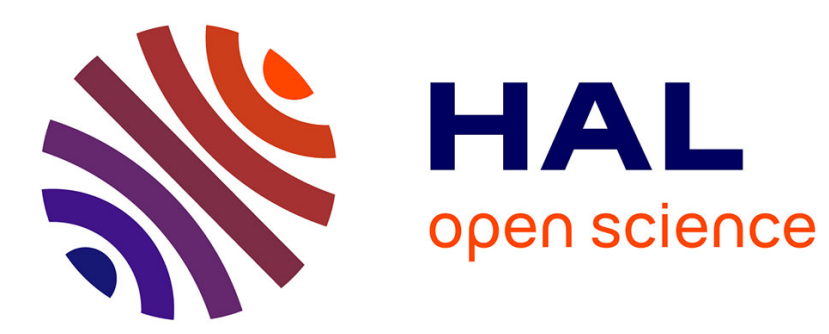

\title{
EADS, une transition inachevée. Une lecture par les catégories de la proximité
}

Damien Talbot

\section{To cite this version:}

Damien Talbot. EADS, une transition inachevée. Une lecture par les catégories de la proximité. Géographie, Économie, Société, 2008, 10 (2), pp.243-261. 10.3166/ges.10.243-261 . hal-02364242

\section{HAL Id: hal-02364242 \\ https://hal.science/hal-02364242}

Submitted on 14 Nov 2019

HAL is a multi-disciplinary open access archive for the deposit and dissemination of scientific research documents, whether they are published or not. The documents may come from teaching and research institutions in France or abroad, or from public or private research centers.
L'archive ouverte pluridisciplinaire HAL, est destinée au dépôt et à la diffusion de documents scientifiques de niveau recherche, publiés ou non, émanant des établissements d'enseignement et de recherche français ou étrangers, des laboratoires publics ou privés. 


\title{
EADS, une transition inachevée
}

\section{Une lecture par les catégories de la proximité}

\author{
EADS, an unfinished transition \\ An understanding by the categories of proximity
}

\author{
Damien Talbot \\ Maître de conférences \\ GREThA \\ (UMR CNRS 5113) \\ Université Montesquieu Bordeaux IV \\ Avenue Léon Duguit 33608 PESSAC CEDEX \\ Tél. : $0556848671 \quad$ Fax. : 0556848647 \\ damien.talbot@u-bordeaux4.fr
}

in

Géographie, Economie, Société, 2008, vol. 10, n², pp. 243-261

\begin{abstract}
Résumé
Le groupe aéronautique européen EADS a rencontré de nombreux problèmes en 2006. Les analyses convergent pour y voir un «problème de gouvernance ». Ce constat ne suffit pas. Nous proposons deux grandes explications complémentaires : ces difficultés de gouvernance résultent d'une trop grande distance, à la fois d'ordre géographique et social. Nous utilisons une définition de la proximité dotées de dimensions spatiale et non-spatiale, pour développer notre argumentation. Au côté de la proximité géographique, nous distinguons la proximité institutionnelle et la proximité organisationnelle. La première est comprise comme une disponibilité relationnelle, la deuxième comme le fait de se conformer et de partager des institutions, la troisième comme une forme particulière de la précédente qui articule coordination politique et coordination cognitive. Les difficultés d'EADS sont alors principalement interprétées comme le résultat d'un défaut de proximité institutionnelle.
\end{abstract}

Mots clés : proximités, institutionnalisme américain, secteur aérospatial, EADS.

\begin{abstract}
Aeronautical group European EADS had many difficulties in 2006, interpreted as "problem of governance". This interpretation is not sufficient. We propose two complementary explanations: these difficulties of governance result from a too long distance, at the same time of a geographical and social nature. We use a definition of proximity with spatial and non spatial dimensions to develop our argumentation. With geographical proximity, we distinguish institutional proximity and organisational proximity. First is understood as a relational availability, second as conforming and sharing institutions, third as a particular form of the preceding one which articulates political coordination and cognitive coordination. Difficulties of EADS are mainly interpreted as the result of a lack of institutional proximity.
\end{abstract}

Key words: proximities, American institutionalism, aerospace sector, EADS. 


\title{
EADS, une transition inachevée
}

\author{
Une lecture par les catégories de la proximité
}

\section{Introduction}

2006 fut une année difficile pour le groupe aéronautique européen EADS $^{1}$ : sur le plan productif, annonce de nouveaux retards de livraison pour le programme A380; sur le plan des alliances, échec du raid sur Thalès qui s'associe à Alcatel dans les satellites sans EADS ; sur le plan managérial, soupçons de délit d'initiés, succession de changements à la tête du groupe $^{2}$; sur le plan commercial, échec du lancement de l'A350 publiquement critiqué par les compagnies aériennes et remplacé par un programme plus ambitieux mais plus coûteux, l'A350 XWB, et reprise par Boeing de son leadership dans le domaine des ventes (en volume et en valeur) d'avions civils de plus de 100 places $^{3}$; sur le plan financier, premières pertes comptables attendues pour Airbus SAS. Les analystes voient comme fait générateur à ces difficultés un "problème de gouvernance ». Les conflits de personnes, une incompatibilité entre les systèmes électriques des tronçons de l'A380 à l'origine des retards de livraison qui dénotent une insuffisante harmonisation des outils de conception, la présence de quelques « bizarreries organisationnelles » à commencer par une présidence bicéphale ou le principe de « reporting croisé » que nous aurons à décrire, sont autant de signes de ces difficultés.

Mais ce constat d'une mauvaise gouvernance ne suffit pas. Ou plutôt, comment expliquer le paradoxe suivant : pourquoi une entreprise capable de concevoir, de produire et de vendre des produits d'une extrême complexité ${ }^{4}$ rencontre-t-elle de telles difficultés à respecter les principes de base d'une coordination réussie, à savoir l'uniformisation des outils de conception et de production et une structure hiérarchique claire, non redondante ? Nous proposons, et c'est l'objet de cet article, deux grandes explications complémentaires : ces difficultés résultent d'une insuffisance de proximité, à la fois d'ordre géographique et social.

Afin de définir ce que nous entendons par "proximité », nous utiliserons le cadre conceptuel développé par le groupe de chercheurs «Dynamiques de Proximité » ${ }^{5}$ qui cherche à rendre compte des conditions d'ordre spatial et non spatial de la coordination. Trois catégories de proximités sont proposées. La proximité géographique traduit la distance géographique entre deux entités et repose sur une conception enrichie de l'espace, ce dernier étant saisi comme une construction active de relations et non comme un simple contexte fonctionnel. La proximité présente aussi une dimension non spatiale, de type relationnel, puisque l'agent est présent à la fois « ici et ailleurs » (Rallet, 1999). Ici car il est localisé dans un espace géographique au sein duquel il entretient des relations de voisinages, ailleurs car l'acteur est

\footnotetext{
${ }^{1}$ European Aeronautic Defence and Space Company.

${ }^{2}$ Depuis le début 2005, trois coprésidents et un président français se sont succédés.

${ }^{3}$ Boeing a enregistré 1044 commandes contre 790 pour Airbus. Ce résultat constitue toutefois pour l'avionneur européen son second plus haut niveau de commandes, après les 1002 avions vendus en 2005.

${ }^{4}$ EADS rivalise avec Boeing dans le domaine des avions civils (Airbus), est le leader mondial dans la conception et fabrication d'hélicoptères (Eurocopter), fabrique des satellites (EADS Astrium), le lanceur Ariane (EADS Space Transportation), des avions de combat (Eurofighter $\mathrm{GmbH}$ ) et divers missiles (MBDA).

${ }^{5}$ Pour les publications les plus récentes sur ce point, le lecteur peut se référer à Gilly et Torre (2000), Dupuy et Burmeister (2003), Pecqueur et Zimmermann (2004), Talbot et Kirat (2005) ou encore Torre et Rallet (2005).
} 
évidemment en relation à distance avec d'autres agents. Nous distinguons deux formes de proximité non spatiale ${ }^{6}$ : la proximité institutionnelle et la proximité organisationnelle.

Ces trois formes de proximités sont tour à tour définies puis mobilisées pour expliquer les difficultés rencontrées par EADS. Ainsi, et sur un plan géographique, il faut constater l'éclatement spatial des sites de production et de conception d'EADS, puisqu'on en dénombre une cinquantaine au moins en Europe (section 1). Si la faible proximité géographique entre les sites ne facilite certes pas la coordination, il ne faut pas y voir la principale raison aux difficultés rencontrées par EADS. A cette distance physique s'ajoute en effet une distance d'ordre relationnel. La proximité institutionnelle est à la fois composée d'une logique issue de l'histoire de l'industrie aérospatiale, qui sous-tend les actes de coordination, qualifiée d'arsenal (Muller, 1988) et d'une logique de marché relativement plus récente (section 2). A cette proximité composite, il faut associer une proximité organisationnelle qui dans ses fonctions de coordination cognitive pallie à la dispersion géographique, mais dans ses fonctions de coordination politique échoue partiellement du fait de la persistance de deux logiques parfois contradictoires précitées (section 3).

\section{Une faible proximité géographique entre les unités d'EADS}

Nous définissons ici la forme la plus intuitive de la proximité, dite géographique, qui aborde la question des conditions objectives de localisation (Pecqueur et Zimmermann, 2004).

\subsection{La proximité géographique : une disponibilité relationnelle}

Elle est une représentation portée par des individus de la distance qui sépare dans l'espace des objets et/ou des individus. Comme jugement porté sur une distance, elle est doublement relative (Torre et Rallet, 2005) en raison des caractéristiques propres à l'espace géographique :

- $\quad$ relative car la distance est pondérée par le temps et les coûts de transports, du fait de la structuration matérielle de l'espace. Nous renvoyons ici à l'idée classique selon laquelle l'espace physique est structuré par des infrastructures de transport et de communication, constituant un cadre matériel favorisant la circulation des informations, des biens physiques et des individus ;

- $\quad$ relative car la distance qui sépare des individus, des organisations ou des villes est aussi une représentation, un jugement conduisant à se situer de façon binaire "près de» ou «loin de ». Parce que les acteurs co-localisés partagent un certain sens commun du fait d'une localisation identique, les individus qui habitent un même lieu ont de facto une même référence. L'espace géographique ne doit pas uniquement s'entendre comme un contexte purement physique doté d'attributs matériels au sein duquel se déroulent des relations économiques. L'espace est aussi porteur d'une signification intersubjective, au sens de Descombes (1996). Les significations intersubjectives correspondent aux consensus entre des individus indépendants. C'est par exemple le cas des similitudes entre divers jugements de goûts. Il est ainsi possible de constater que les consommateurs ont tendance à adopter tel ou tel modèle

\footnotetext{
${ }^{6}$ Si la définition de la proximité géographique fait l'objet d'un consensus au sein du groupe «Dynamiques de Proximité », il n'en est pas de même du versant relationnel de ce concept. Un premier courant qualifié d'interactionniste se limite à deux formes de proximité (géographique, organisée), tandis qu'un second d'inspiration institutionnaliste décline la proximité en trois composantes (géographique, institutionnelle, organisationnelle) (Gilly et Lung, 2004).
} 
de consommation, tel ou tel type de loisirs, etc., exprimant les mêmes préférences. Cette similitude des jugements peut s'expliquer par les conditions historiques (éducation, action publicitaire, recherche d'un consensus, etc.). Il n'en reste pas moins qu'il s'agit d'une libre rencontre de jugements indépendants, reposant sur «un universel de ressemblance " selon les termes du philosophe (ibid., p. 292). Dans notre cas, la similitude de jugement porte sur le nom de cet espace, ses limites physiques, son histoire, son patrimoine, les usages et les modes de vie qui y prennent place, les coordinations précédentes réussies ou échouées, etc. L'espace géographique, porteur d'une telle signification, devient alors un lieu particulier. Il constitue un référent cognitif et il intervient dans le processus de construction (ou de destruction) des identités dans le sens où il est une composante du rapport aux autres (Pecqueur, 1997), puisque les acteurs locaux le font exister au regard des autres (Laganier, Villalba et Zuindeau, 2004).

Le fait de se sentir " proche » ne doit pas être interprété comme l'assurance qu'une relation s'instaure. Les significations intersubjectives faisant l'objet d'un consensus sont aisément communicables, mais la relation à autrui ainsi posée n'est ici qu'une possibilité de relation entre deux individus pour l'instant indépendants. Cette ressemblance ne repose sur aucune connexion positive des individus et n'implique pas une relation systématique, même si elle permet de prévoir le type de liens envisageables. La même remarque peut être faite au sujet de la structuration matérielle de l'espace : une route n'implique pas une interaction. Cela signifie simplement que l'existence d'une forte proximité géographique entre acteurs n'est pas neutre : elle est une mise en disponibilité relationnelle, une ressource encore latente ${ }^{7}$. Par conséquent, sa faiblesse, comme dans le cas d'EADS, n'est pas un problème insurmontable à condition d'élaborer des outils de coordination compensant la dispersion géographique.

\subsection{L'éclatement dans l'espace européen des sites d'EADS}

EADS, et tout particulièrement sa filiale Airbus, sont le résultat de consolidations d'abord infranationales, puis internationales. Le groupe européen hérite donc d'une grande quantité de sites. A cet aspect quantitatif s'ajoute le fait que la géographie de l'industrie aéronautique se caractérise par une grande inertie depuis la création des premiers établissements. Cette stabilité avait suscité la création de bassins spécialisés où, autour des sites d'assemblage des avionneurs, gravitait un vaste tissu de sous-traitants spécialisés (Beckouche, 1996). Dans le cas français, et dans le cadre d'une logique d'arsenal (cf. infra), l'Etat actionnaire faisait appliquer aux industriels sa politique d'aménagement du territoire, interdisant dès lors tous regroupements de sites pouvant induire des fermetures. En 2006, hors bureaux commerciaux et supports clients, nous dénombrons en Europe pas moins de 51 sites de conception et fabrication $^{8}$, dont 16 appartiennent à Airbus SAS (Carte $\left.n^{\circ} 1\right)$.

\section{Carte 1 Les principaux sites de production d'Airbus SAS en 2006}

\footnotetext{
${ }^{7}$ Cette possibilité de relation ne préjuge en rien de son contenu, dans le cas où elle se noue. La littérature sur les conflits d'usage de l'espace ( $c f$. notamment Caron et Torre, 2005 ; Jeanneaux et Kirat, 2005) cite à ce propos nombre d'exemples d'inégalités liées à la situation topographique des acteurs (un usager subit la pollution de l'eau provenant d'un autre usager situé en amont). Elle démontre que la proximité géographique, quand elle est subie, peut être constitutive d'inégalités créant des rapports de force entre les acteurs.

${ }^{8}$ Sièges sociaux inclus.
} 
Les différentes pièces d'un Airbus sont fabriquées aux quatre coins de l'Europe : les ailes au Royaume-Uni, le fuselage en France, la cabine intérieure en Allemagne, l'empennage horizontal en Espagne, etc. (Fig. 1). Cette dispersion induit d'importants flux logistiques générateurs de surcoûts.

Figure 1 Le transport (simplifié) des éléments de l'A380 par voies maritime, aérienne et terrestre

On le voit, à condition d'élaborer des outils de coordination (ici logistique, cf. infra pour des outils permettant une coordination cognitive) qui compensent cette dispersion, la proximité géographique n'est pas un élément indispensable à la production d'un produit complexe. En revanche, cette proximité peut s'avérer utile, de façon temporaire, lors des phases de conception de l'appareil.

\subsection{La proximité géographique temporaire pour concevoir ensemble : les équipes plateaux d'Airbus}

Nous distinguons ici les besoins permanents de proximité géographique (impliquant la colocalisation) et les besoins temporaires qui ne concernent que certaines phases d'une activité ou qui s'exprimeront à différents moments du processus productif. Pour qualifier ces besoins, on parle de proximité géographique temporaire (impliquant la co-localisation temporaire), celle-ci pouvant être assurée par la délégation de personnels ou l'organisation de réunions (Coris et Lung, 2005). Dans ce cas, ce n'est pas tant le partage de significations intersubjectives générées par une co-localisation dans un lieu particulier qui est utile aux activités de conception, que le face à face qui est facilité par un espace structuré physiquement.

Le face à face en effet, tout particulièrement dans les phases de conception commune, demeure essentiel pour répondre à des questions techniques et productives nouvelles, car il permet de fluidifier les relations et de rendre les discussions plus interactives (Rallet et Torre, 2007). Il facilite grandement la recombinaison des compétences dispersées en permettant le transfert de connaissances, tout en réduisant l'incertitude issue de la dimension tacite inhérente à tout savoir et les risques d'opportunisme (Boschma, 2005). Il en est ainsi de la constitution d'équipes plateaux en conception qui suppose une proximité géographique temporaire entre les salariés des différents sites d'Airbus. L'existence d'une telle proximité géographique est parfaitement illustrée par le cas toulousain'. C'est à l'occasion du développement de l'A340-500/600 en 1997 qu'Airbus a développé sa première équipe plateau. L'A380 approfondit cette organisation avec la construction d'un plateau d'un millier d'ingénieurs et de techniciens à Toulouse où sont réunis les personnels de l'avionneur, mais aussi des équipementiers de premier rang. Ce besoin intense de face à face, auquel répond l'équipe plateau, concerne uniquement les phases de conception générale de l'avion et de développement pour mettre au point les solutions techniques retenues. Cette période de quelques mois est l'occasion de recombiner des compétences dispersées entre différents acteurs éloignés physiquement en vue d'innover et de résoudre un problème productif. Le plateau est ensuite dissout, l'ingénierie simultanée permettant sa virtualisation. Il s'agit de remplacer, quand cela est possible, les rencontres physiques par d'autres types d'interactions à distance.

\footnotetext{
${ }^{9}$ Pour un retour sur la trajectoire du complexe aéronautique toulousain, $c f$. pour les contributions les plus récentes Hickie (2006), ou encore Leriche et Zuliani (2007).
} 
De façon plus générale, la proximité géographique n'apparait pas, à elle seule, comme une condition permissive suffisante pour que s'engage une action collective. Cette dernière suppose pour s'établir le partage entre les acteurs d'une proximité que nous qualifions d'institutionnelle.

\section{La proximité institutionnelle dans l'industrie aérospatiale : une logique de marché dominante, une logique d'arsenal persistante}

Avant de rendre compte de la proximité institutionnelle qui permet aux acteurs de l'industrie aérospatiale d'agir ensemble, nous proposons une définition de ce terme.

\subsection{La proximité institutionnelle : partager et se conformer à des institutions}

Le concept d'institution proposé par les institutionnalistes américains, et sur lequel nous faisons reposer notre définition de la proximité institutionnelle, renvoie à deux idées centrales: ne pas séparer la cognition de l'action, l'institution relevant des deux niveaux simultanément ; souligner que l'institution, comme acte collectif, est au service d'une finalité. Développons ces deux idées.

L'unité des institutionnalistes américains se fait sur la nécessité de rejeter le dualisme cartésien entre pensée et action, suivant en cela la philosophie pragmatique développée notamment par C.S. Pierce. Chez cet auteur, les idées ne sont plus des a priori hors du temps, mais des significations produites et reproduites dans un processus d'interaction constant entre la pensée des faits et l'expérience des faits (Bazzoli et Kirat, 2003). Cette conception réunifie le monde de la pensée et le monde des actes. Elle invalide la frontière traditionnelle proposée entre pensée et action, pour en venir à une distinction entre un monde naturel et un monde institutionnel.

Par ailleurs, les institutions sont préétablies, non seulement par des individus à leur propre intention, mais elles sont aussi socialement préétablies. Autrement dit, elles nous prééxistent et s'imposent à nous. En ce sens, elles constituent des attentes normatives définies préalablement à tout exercice et doivent être considérées comme des prêts-à-penser et prêts-àfaire dont les individus ne sont pas a priori les auteurs (Descombes, 1996). Cela signifie que la dimension téléonomique, l'orientation vers une fin, est une donnée majeure des institutions. Elles sont porteuses d'une "intentionnalité collective»(Searle, 1998) qui transcende les intentionnalités individuelles (Bessy, 2002). Elles sont des artefacts sociaux, profondément humains et ont un caractère performatif qui leur assure, d'ailleurs, une certaine pérennité. Comme les actions ne sont ni neutres ni objectives mais instituées, elles sont construites en fonction des finalités et doivent d'être examinées en référence à ces mêmes fins.

Dans cette double perspective, l'institution est à la fois la règle et le comportement, les représentations et les pratiques, les habitudes de penser et de faire, sans que les uns ne soient réductibles aux autres. Toute action devient une sorte de langage, traduisant une idée, la croyance se manifestant dans la conduite. Il s'agit en quelque sorte de " faire » des idées collectives en vue d'atteindre un objectif. L'institution devient alors une idée commune en acte au service d'une finalité.

Ce que «dit» ce langage sont des idées communes à un groupe d'acteurs. Pour qu'un ensemble d'acteurs puisse mobiliser une même institution, il est indispensable que les participants aient la même représentation de ce qu'ils sont en train de faire. Ils partagent, alors, des significations publiques et sociales, qui ne sont pas identiques par coïncidence, mais «sont inculquées aux individus de façon à rendre possible, de la part de chacun d'eux, des 
conduites coordonnées et intelligibles du point de vue du groupe » (Descombes, 1996, p. 294). Elles sont constitutives d'une forme de proximité nommée institutionnelle, qui toutefois ne s'y réduit pas.

Ces significations communes présentent en effet un côté structural. Elles ne mettent pas en présence deux individus sans liens, mais "deux partenaires qui doivent faire des choses différentes et dont les rôles et les statuts sont justement fixés par une règle établie, un usage social que les gens suivent » (Descombes, 1996, p. 297). Par exemple, la propriété suppose un système de relations sociales qui affecte des statuts particuliers aux détenteurs des droits de propriété et organise les relations avec les autres acteurs (autres propriétaires, locataires, salariés, etc.). Par conséquent, la proximité institutionnelle, comme condition de l'action collective, ne peut renvoyer à la seule existence de significations communes aux acteurs. L'institution génère un autre élément de la proximité institutionnelle, relatif au constat de la fixation par l'institution de rôles complémentaires à jouer dans l'action collective par des acteurs. Nous comprenons ici en quoi la proximité institutionnelle est irréductible à une logique de similitude. Cette proximité n'est pas du seul ordre de la ressemblance ${ }^{10}$ fondé sur une relation entre deux faits et/ou individus distincts et indépendants. Il faut y ajouter un ordre de la communauté qui relie deux faits et/ou individus désormais dépendants, constitués en système. On passe alors d'une ressemblance de caractère à une connexion positive.

Il faut ajouter que la complémentarité des rôles des partenaires est intrinsèquement génératrice d'inégalités. Autrement dit, la relation sociale n'est pas a priori équitable et juste. Il s'agit au contraire d'un rapport de force a priori, ce qui explique pourquoi cette relation est par nature conflictuelle. "Les institutions peuvent incorporer des rapports de force et pas seulement une vision du monde partagée » (Amable et Palombarini, 2004, p. 32). D'autant que les partenaires n'émergent pas ex nihilo afin de se coordonner : il existe une asymétrie de ressources cognitives et matérielles issues de l'histoire des agents, d'une position sociale, ce qui rend ex ante les termes de l'échange plus favorables à l'une des parties. Cela signifie que les conflits inhérents aux hiérarchies sociales doivent être, toujours transitoirement, régulés, voire apaisés. C'est une condition à l'action collective. La proximité institutionnelle, comme « réalisation des conditions » (Pecqueur et Zimmermann, 2004, p. 25) de l'action collective ne doit pas être réduite à une seule dimension cognitive renvoyant à une vision partagée du monde ; il faut y associer une dimension politique régulatrice qui attribue des rôles à des acteurs hétérogènes afin d'apaiser transitoirement les conflits.

$\mathrm{Au}$ final, mobiliser une institution revient à entrer potentiellement ou effectivement en relation inégale avec autrui, auprès duquel on est dorénavant proche. Cette proximité institutionnelle désigne le fait pour un ensemble d'individus de partager et de se conformer à des idées communes et à des rôles fixés par des institutions. Ces individus " font», en adoptant des rôles complémentaires, des idées communes.

Pour rendre compte de la proximité institutionnelle qui existe entre les acteurs de l'industrie aérospatiale, nous distinguons deux périodes qui ponctuent l'histoire de ce secteur d'activité : la première, qui va du début du $\mathrm{XX}^{\mathrm{e}}$ siècle au milieu des années soixante-dix, voit les relations entre les acteurs du secteur régies par un ensemble d'institutions partagées qualifiées de logique d'arsenal. Depuis une quarantaine d'années, cette dernière est progressivement remise en cause au profit d'une autre logique de proximité, dite logique de marché. Cela ne signifie pas pour autant que la logique d'arsenal a totalement disparu. Nous comprenons alors la

10 Descombes (1996) en donne un bon exemple : participer à une négociation collective, c'est partager avec tous les participants un certains nombres de croyances relatives au dialogue social. Cela n'implique pas une similitude d'opinions ou d'intérêts. L'exemple vaut aussi pour la participation à une élection. 
proximité institutionnelle comme une combinaison de deux logiques, de marché et d'arsenal, la première dominant la seconde.

\subsection{La proximité institutionnelle historique : la logique d'arsenal}

Cette proximité institutionnelle est issue de l'histoire de l'industrie aérospatiale, qui prend son essor au début du $\mathrm{XX}^{\mathrm{e}}$ siècle. Elle se fonde sur l'idée communément partagée selon laquelle la construction d'un avion constitue une occasion de développer des technologies, sources de prestige et d'indépendance nationale. L'Etat cherche à maîtriser ce secteur vital pour la nation et en constitue dés lors l'acteur clé.

\subsubsection{L'idée commune fondatrice : la technologie pour elle-même}

Les acteurs de l'industrie aérospatiale sont porteurs, au moins au départ, de plusieurs idées communes constitutives d'une logique d'arsenal. Ainsi, selon une telle logique, un avion efficient est un avion performant techniquement (à l'exemple du Concorde, avion d'ingénieurs par excellence), l'aspect technologique étant prédominant, tandis que les nécessités commerciales sont reléguées au second plan. Dans l'esprit de tous, l'Etat se doit d'être l'acteur central de cette industrie, pour au moins trois raisons issues des caractéristiques de l'industrie aérospatiale. Premièrement, l'aérospatial est un secteur dit de «frontière technologique » dans lequel tout pays industrialisé aspirant à un certain rang mondial se doit de figurer. Deuxièmement, ce secteur provoque des retombées bénéfiques à l'ensemble de l'industrie nationale. Troisièmement, le caractère militaire d'une partie de l'industrie aérospatiale conduit l'ensemble de cette dernière à être placé sous tutelle étatique.

\subsubsection{Le rôle prédominant de l'Etat : à la fois client, actionnaire et financeur}

Dès lors, l'Etat définit une politique technologique afin de conserver, voire de développer, un niveau technologique auquel les seules forces d'un marché trop étroit ne peuvent conduire. Les industriels sont dans l'attente de décisions gouvernementales pour agir. Depuis le début du XX ${ }^{\text {ième }}$ siècle, l'Etat désigne le (ou les) constructeur(s) et indique les caractéristiques de l'avion à construire. Il est client et actionnaire des entreprises qui réalisent les programmes, dont il est le premier financeur.

Dans un tel contexte de préservation de l'indépendance nationale, les coopérations européennes doivent se réaliser sur le mode égalitaire et par programme. Dans le cas d'une coopération bipartite, cela signifie que les coûts, les charges de travail induites ainsi que les bénéfices éventuels sont divisés à parts égales. Les raisons de la mise en place de telles coopérations sont multiples : mise en commun de savoir-faire, de technologies, d'un potentiel industriel, d'un marché domestique, ou encore partage des coûts et des risques, mais aussi sur un plan plus politique participation à la construction de l'Union Européenne.

Progressivement, cette logique est de moins en moins acceptée par les industriels en raison des échecs commerciaux plus ou moins sévères qu'ils connaissent (à l'instar du programme Concorde). En réponse, certains dirigeants de l'époque vont chercher à dépasser la logique d'arsenal et proposent une nouvelle organisation industrielle et commerciale des coopérations internationales, en créant par exemple le GIE ${ }^{11}$ Airbus en 1971.

\footnotetext{
${ }^{11}$ Groupement d'Intérêt Economique.
} 


\subsection{Une nouvelle proximité institutionnelle: une logique de marché qui s'affirme progressivement, même si la logique d'arsenal persiste encore}

Dans une logique de marché, les préoccupations commerciales prennent peu à peu le pas sur les seules considérations politiques. L'idée selon laquelle un avion doit correspondre aux besoins des compagnies aériennes s'affirme, ce qui renforce le rôle des industriels détenteurs des compétences commerciales.

\subsubsection{L'idée commune fondatrice : un bon avion est un avion qui se vend}

Un programme est perçu comme une réussite si l'appareil se vend. Il faut voir là un basculement des priorités : la technologie ne doit être utilisée que si elle confère au produit un avantage commercial, et non plus pour elle-même. Ce n'est donc pas à un rejet des techniques auquel nous assistons, mais plutôt à une réorientation de la finalité de ces dernières qui doivent avant tout favoriser les ventes des appareils. Et dans un marché surtout attentif au prix, les innovations radicales sont abandonnées au profit d'améliorations constantes des produits suivant les demandes des clients. Toutes les améliorations ont pour objectif d'aboutir à une réduction sensible pour les compagnies aériennes des coûts d'achats, d'exploitation et de maintenance. La famille des Airbus (programme A300B lancé en 1968) est ainsi conçue en respectant cette idée : un avion doit être simple et peu cher, correspondant aux besoins du marché.

\subsubsection{Une nouvelle répartition des rôles : des clients prescripteurs, des industriels concepteurs, un Etat financeur}

Dès lors, ce sont les compagnies aériennes consultées qui fixent les caractéristiques et le prix de l'appareil (Beteille, 1995). Ce prix devient d'ailleurs une condition essentielle du succès d'un programme conçu entièrement par les industriels. Au même moment, l'Etat cherche à réduire son rôle dans cette industrie afin de minimiser ses dépenses dans le contexte de crise des années soixante-dix. On assiste alors au retrait progressif de l'Etat autorisant une émancipation des industriels. Dans une logique d'arsenal, nous l'avons dit, l'Etat est tout à la fois client, actionnaire et financeur des industriels. Dans une logique de marché, il conserve principalement ce dernier rôle. Le maintien du soutien public à l'aéronautique trouve son origine dans les coûts et les risques très importants liés au lancement de nouveaux produits.

Dans ce contexte, les coopérations internationales multipartites ne fonctionnent plus sur un mode égalitaire comme ce fut le cas dans le passé. Les règles de compensations industrielles imposées par les Etats et d'attribution de charges de travail en fonction de critères politiques, disparaissent peu à peu. Dans le cas d'Airbus, afin de maximiser les compétences et d'éviter les duplications fonctionnelles, les partenaires sont cantonnés dans une spécialité par le groupe, qui répartit la charge industrielle : les coopérations deviennent alors inégalitaires. Il s'agit là d'une explication à la spécialisation croissante des sites Airbus (cf. infra).

\section{La proximité organisationnelle au sein du groupe EADS : une concrétisation de la logique de marché}

Sur la base de cette proximité institutionnelle, des relations complexes, délimitées et orientées deviennent possibles entre de multiples individus. Il en est ainsi des coutumes, des habitudes collectives, des préjugés, des règles juridiques ou morales mais aussi des parlements, des entreprises, des associations, etc., qui constituent une manifestation concrète des institutions. 
Ce sont tous des phénomènes fondamentalement de même nature, de l'ordre de l'action sociale. Ces phénomènes ne sont pas pour autant parfaitement identiques et diffèrent, selon les institutionnalistes américains, en degré d'organisation. Il est nécessaire d'approfondir ces différences, tant le concept d'organisation occupe une place importante dans les sciences sociales. Pour Mitchell (1935), l'institution peut être inorganisée (coutume) ou organisée (famille, entreprise, ou encore l'Etat). Dans une perspective veblenienne, où les institutions régissent à la fois les rapports entre les individus mais aussi entre les individus et le monde matériel, l'organisation apparaît comme une solution institutionnelle à une contrainte technologique (Palloix, 2000). Chez Commons (1934), l'institution peut prendre la forme d'organisations actives (going concerns) qui se définissent par un but, des règles de fonctionnement (working rules) et des autorités qui formulent et appliquent ces mêmes règles. Dans ce cadre conceptuel, le terme «organisation» a vocation à rendre compte des institutions qui présentent un caractère organisé. Suivant cette distinction, nous comprenons la proximité organisationnelle comme une forme de proximité institutionnelle.

\subsection{La proximité organisationnelle : une forme particulière de proximité institutionnelle}

La proximité organisationnelle "lie les agents participant à une activité finalisée dans le cadre d'une structure particulière. (...) [Elle] se déploie à l'intérieur des organisations (firmes, établissements, etc.) et, le cas échéant, entre organisations liées par un rapport de dépendance ou d'interdépendance économique ou financière (entre sociétés membres d'un groupe industriel ou financier, au sein d'un réseau, etc.) (Kirat et Lung, 1995, p. 213). Ainsi, l'individu qui intègre une organisation partage de fait une proximité organisationnelle avec l'ensemble des membres. Il s'agit toujours de partager un espace social qui prend cette fois-ci une forme concrète observable par tous (à la différence par exemple d'une coutume). Reste à donner un contenu à cette proximité organisationnelle.

Les organisations ont vocation à permettre une action collective complexe en coordonnant cognitivement et politiquement les actions individuelles. Elles vont mettre en actes des idées communes en vue d'atteindre un objectif, ce qui suppose la résolution de nombreux problèmes de coordination. Sur un plan cognitif, les organisations sont des institutions qui ont la particularité de produire et d'activer des règles et des routines qui assurent une coordination de nature cognitive répondant à la problématique de l'efficacité des actions. Il s'agit, par les règles, de réduire l'incertitude et de sécuriser les anticipations. Quant aux routines, par leurs caractères mécaniques et automatiques, elles suspendent en effet l'incertitude liée à l'action de l'autre, et apparaissent comme une stabilisation de l'interprétation des règles car elles « (...) sont un mode de résolution pragmatique d'un problème auquel les règles sont une réponse théorique (...) » (Reynaud, 2001, p. 64). Les routines et les règles sont autant de ressources cognitives qui vont faire l'objet d'un apprentissage de la part des membres de l'organisation. Il existe alors un processus d'accumulation de telles ressources en vue de réaliser des tâches de manière efficace et qui correspond à la construction d'une mémoire organisationnelle.

Les organisations, comme formes institutionnelles, ne doivent pas être réduites à une seule fonction de coordination cognitive. Les institutionnalistes affirment en effet que l'action exprime des choix en relation avec des phénomènes, certes de signification et d'évaluation, mais aussi de pouvoir (Théret, 2001). La pensée institutionnaliste, en mettant l'accent sur l'artificialité de l'action et sur l'importance des rapports de pouvoir et des conflits qui en découle, permet d'ailleurs de rappeler que le partage peut résulter tout autant de rapports de coercition. Sur un plan politique, la production de règles et de routines par les organisations répond ici à la problématique de la conformité et de la légitimité des actions réalisées par des 
acteurs hétérogènes. Dit autrement, la coordination politique ${ }^{12}$ implique de prendre des décisions par nature partiales tout en donnant une cohérence à des aspirations contradictoires. Il est possible caractériser les organisations de la façon suivante (Bazzoli et Dutraive, 2002) : pour exister, une organisation doit avoir une constitution juridique qui renvoie aux buts de celle-ci et exprime les relations de pouvoir et d'autorité. Elle repose sur des règles externes, des règles de droit qui fondent l'existence légale de l'organisation en déléguant un pouvoir normateur et de mobilisation des ressources à ses représentants. Elle repose aussi sur des règles internes, qui organisent l'exercice du pouvoir, définissent les termes de l'appartenance à l'organisation et permettent la réalisation de choix collectifs. En outre, une organisation est une institution qui perdure dans le temps, malgré le changement de ses membres.

Au final, nous définissons les organisations comme des institutions dotées :

- d'une mémoire composée de règles et de routines comprises comme autant de ressources cognitives mobilisées pour la mise en cohérence des actions individuelles et pour atteindre efficacement les objectifs fixés (coordination cognitive) ;

- et d'une structure de pouvoir qui a en charge cette mise en cohérence en incitant/veillant à la conformité de ces actions aux règles produites, ce qui permet leur légitimation et la fixation des choix collectifs (coordination politique).

D'un point de vue théorique, la proximité organisationnelle consistera alors pour des acteurs $a$ priori hétérogènes à intégrer la communauté cognitive, c'est-à-dire à accéder à la mémoire de l'organisation composée de ressources cognitives, et à intégrer la communauté politique, c'est-à-dire à s'insérer dans la structure de pouvoir. Elle est bien une forme particulière de proximité institutionnelle qui consiste, rappelons-le, à partager des idées communes et à assumer des rôles complémentaires. D'un point de vue empirique, EADS, en tant que groupe, constitue une illustration de cette définition. En effet, sur le plan politique, EADS se compose de liens hiérarchiques entre la société mère, constituant le centre de décision, et ses unités (filiales, sociétés contrôlées minoritairement, etc.). Ces structures, qui découlent des propriétés juridiques, sont les vecteurs des relations financières et patrimoniales autorisant plus ou moins d'autonomies financière et stratégique aux unités qui se conforment aux règles édictées par la tête de groupe. Elles ont vocation à réguler les conflits et à fixer les choix collectifs en fonction des intérêts de l'organisation. Sur le plan cognitif, vont se nouer des rapports de production, qui ne sont plus nécessairement verticaux dans le sens où ils peuvent concerner directement des unités entre elles. Ces rapports résultent des contraintes de la division cognitive du travail au sein du groupe. Cette coordination suppose des mécanismes d'apprentissage ayant pour but la création et l'assimilation de règles et de routines, visant dans notre cas à opérer à une rationalisation systémique de l'activité. Cette rationalisation est patente dans le cas d'Airbus. Nous revenons plus en détail sur ces deux types de coordination.

\subsection{La coordination politique : un fragile compromis franco-allemand}

La coordination politique d'EADS présentait jusqu'en 2007 la particularité de reposer sur une structure bicéphale, conduisant à la désignation de deux présidents, tout en intégrant des Etats dans son actionnariat. Il apparaît en outre que la proximité organisationnelle n'est pas

\footnotetext{
12 Pour Amable et Palombarini (2004), le terme «politique » renvoie à un sous-système social dans lequel sont fixés les choix collectifs qui s'imposent aux acteurs sociaux dans leur ensemble. Chaque acteur essaye alors d'obtenir et de mettre en œuvre une capacité d'influence sur les choix.
} 
isomorphe, certaines unités disposant de plus ou moins d'autonomie décisionnelle ou étant plus ou moins bien intégrées.

\subsubsection{Des Etats actionnaires, une structure bicéphale et le reporting croisé}

Les rivalités nationales restent fortes au sein même d'EADS. Cette entreprise est le produit d'un subtil compromis entre les intérêts nationaux allemands et français dans son actionnariat et dans son management.

L'actionnariat est relativement atypique puisqu'il rassemble des acteurs publics et privés, qui plus est de nationalités différentes, dans un secteur traditionnellement peu ouvert aux participations étrangères. Le français Lagardère, l'allemand Daimler Chrysler, les Etats français et espagnols sont les principaux actionnaires de la nouvelle entité ${ }^{13}$. Ainsi, deux acteurs publics participent à la définition de la stratégie du groupe européen. L'Etat français conserve en outre le contrôle des unités d'EADS qui ont en charge la réalisation des missiles balistiques constitutifs de la force de dissuasion nucléaire française, et un droit de veto triple pour les alliances stratégiques, les augmentations de capital et les restructurations industrielles.

Le management est encore plus atypique. Au nom de la parité, un système à double commande franco-allemande avait été instauré à la tête d'EADS. Il fallait voir dans cette exception une traduction organisationnelle concrète de la logique d'arsenal. Ce compromis fragile a été périodiquement remis en cause ${ }^{14}$, jusqu'à l'abandon de la présidence bicéphale en juillet $2007 \mathrm{du}$ fait d'une intervention politique commune aux gouvernements allemand et français. Autre exception encore d'actualité cette fois, l'existence d'un système de « reporting croisé » : le dirigeant français d'une filiale dépend d'un dirigeant allemand de la maison mère et réciproquement. A titre d'exemple, le président du Conseil d'Administration d'EADS est allemand, le directeur général d'EADS (Chief Executive Officer) qui dirige le groupe est français, la direction de trois Divisions sur cinq échouant à des allemands. Cette duplication et ce marquage entre les deux pays se retrouvent à tous les autres niveaux. Les nominations de responsables combinent l'impératif politique et la compétence industrielle, hybridant logique d'arsenal et logique de marché. Cette organisation induit alors d'importantes pesanteurs. D'autant que quatre niveaux de responsabilité sont observables entre l'actionnariat et la gestion opérationnelle des divisions, ce qui complexifie encore la coordination, multipliant les incompréhensions et les occasions de conflits.

Finalement, dans le respect de la logique d'arsenal, l'industrie aéronautique apparaît toujours comme un outil au service de la préservation de la souveraineté nationale : la présence d'Etats dans l'actionnariat d'EADS et le système de reporting croisé ont vocation à permettre à chaque partenaire de peser sur les décisions, d'orienter la stratégie du groupe dans le sens de la préservation de leurs intérêts nationaux jugés essentiels.

\footnotetext{
${ }^{13}$ En 2006, l'actionnariat d'EADS se compose de la façon suivante : actionnariat flottant 42,09\%; Sogeade (50\% Lagardère, 50\% Etat français) 29,96\% ; DaimlerChrylser 22,47\% ; Sepi (Etat espagnol) 5,48\% ; salariés $1,04 \%$; Etat français $0,06 \%$. Les deux actionnaires de référence que sont Daimler Chrysler et Lagardère ont entériné leur prise de recul au printemps 2006, lorsqu'ils ont annoncé qu'ils s'apprêtaient à céder chacun 7,5\% du capital d'EADS. Simultanément, la banque d'Etat russe Vnejtorbank a acheté $5 \%$ du capital. Face à cette fragilisation du contrôle, les Etats espagnol et allemand affichent leur volonté de monter au capital d'EADS.

${ }^{14}$ Il suffit pour s'en convaincre d'examiner les luttes d'influences qui ont débuté au premier semestre 2005 pour le renouvellement de l'équipe dirigeante, pour se terminer fin 2006. Les actionnaires allemands ont estimé à cette époque que leurs homologues français tentent d'abandonner la structure bicéphale d'EADS pour une présidence unique française.
} 


\subsubsection{Une proximité organisationnelle polymorphe déformée par les rapports de pouvoir}

EADS a été construit tout naturellement autour de l'ex GIE Airbus et ce pour plusieurs raisons. Le GIE fut la forme de coopération industrielle et commerciale la plus aboutie en Europe. Aerospatiale, Dasa et Casa ont ainsi appris à travailler ensemble depuis une trentaine d'année au fil des programmes : l'intégration n'en était que plus facile. Les chances de réussite de la fusion étaient encore accentuées par le fait que les Etats espagnol, allemand et français ont principalement apporté à la société unique européenne des actifs civils, par nature plus aisément mutualisables que les activités militaires. De fait, quel que soit le critère choisi, l'activité issue des programmes Airbus apparaît comme dominante au sein du groupe. En 2006 en effet, $49 \%$ des effectifs appartiennent à la filiale Airbus, qui réalise $64 \%$ du chiffre d'affaires total et $80 \%$ du carnet de commandes.

Sur le plan de la coordination politique, ce déséquilibre pose des difficultés internes tant le groupe est dépendant de sa filiale qui dispose de fait d'une large autonomie décisionnelle (Frigant, Kechidi, Talbot, 2006). Fait exceptionnel, des conflits se sont fait jour dans la presse $^{15}$ en ce qui concerne la définition de la stratégie d'Airbus, la maison mère ayant par exemple des difficultés à imposer ses vues à l'avionneur en terme de localisation des unités de production, la première envisageant une relocalisation sur le territoire américain refusée par l'équipe dirigeante d'Airbus.

Une autre conséquence de cette domination des activités d'Airbus dans EADS est le rejet en périphérie de certaines unités moins bien intégrées (Talbot, 2005). Prenons le cas de l'unité bordelaise du groupe. EADS Space Transportation est un maître d'œuvre spécialisé dans le transport et les infrastructures spatiales. Si la filiale consolidée EADS ST voit sa charge d'activité se répartir équitablement autour des domaines de la Défense, des infrastructures orbitales et d'Ariane, le site de Bordeaux demeure fortement spécialisé dans le domaine militaire ( $85 \%$ du niveau d'activité de l'établissement). Le site girondin n'est donc pas représentatif d'un groupe centré sur les activités civiles, d'autant que, dans le strict respect de la logique d'arsenal, son rôle clé dans la construction de la force de dissuasion française le place de facto sous le contrôle direct de l'Etat français. Si on le compare à ses homologues toulousains (Airbus), l'établissement est doté d'un statut périphérique au sein du groupe. La proximité organisationnelle, loin d'être isomorphe, présente ici des caractéristiques différentes selon les unités concernées. Elle se déforme en fonction des rapports de pouvoir, provenant des asymétries de ressources cognitives et matérielles, qui se diffusent dans une structure organisationnelle complexe. Une façon de réduire cette complexité, en attendant une intégration plus poussée des unités, est d'intensifier la coordination cognitive, l'autre facette de la proximité organisationnelle.

\subsection{La coordination cognitive : une intensification encore insuffisante}

Groupe récemment créé, EADS doit surmonter deux obstacles dans la coordination : gérer une multiplicité de sites porteurs de compétences qui se chevauchent, voire se dupliquent; favoriser la diffusion des routines entre des unités de cultures et de passés différents. La réponse est double : créer un réseau interne de circulation d'information et des résultats de la recherche, poursuivre la spécialisation des sites entamée durant les décennies précédentes par Airbus.

\footnotetext{
${ }^{15}$ Cf. par exemple Le Monde, 12 janvier 2004.
} 


\subsubsection{Diffusion de l'information}

Dans les faits, une part importante de la charge de coordination cognitive a été intégrée dans le «Système de Production Informatisée d'Eléments Regroupés » (SPIDER). Grâce à une codification de chaque pièce, le SPIDER permet une standardisation des processus de conception et de gestion de la production par familles de pièces. Il faut y voir la mise en œuvre du concept de Technologie de Groupe, qui consiste à regrouper des objets (pièces, dessins, postes de travail, compétences, etc.) en familles homogènes pour tirer profit des analogies et de l'expérience de l'entreprise lors de la conception et fabrication des produits (Cohendet, Lerena, Sorge, 1992). Cette démarche a, dès lors, permis de mettre en place un système de transfert intra-organisationnel des compétences et des connaissances. A travers les procédures qu'il a générées, ce système s'avère être un support essentiel de la coordination cognitive interne à l'entreprise. Mais pas uniquement.

Cette rationalisation interne de la gestion de production, à travers la codification et la standardisation, induit une importante densification des interactions externes nécessaires à la recomposition, à l'assemblage de l'avion (Kechidi, 2005). Dès lors, ce système a généré de forts besoins de coordination cognitive entre les différents acteurs intervenant dans la réalisation des produits. Il s'agit de permettre aux sites européens de production d'échanger progressivement des données et d'homogénéiser les méthodes de formation, de support et de documentation. Concrètement, s'est constitué un vaste réseau de liaison informatique (Intranet, EDI, etc.) entre les différents sites du constructeur et même au delà, avec ses principaux fournisseurs. Plus qu'un simple échange de données, ce système fonctionne comme une mémoire partagée constitutive d'une proximité organisationnelle fournissant autant de repères pour le travail en commun.

\subsubsection{Une spécialisation croissante des sites d'EADS}

Dans le respect d'une logique de marché qui suppose une réduction constante des duplications fonctionnelles, le groupe EADS accroît la division cognitive du travail entre ses différents établissements, suivant en cela le mouvement de spécialisation des sites initié en son temps par le GIE Airbus. Ainsi, à partir de 1988, chaque usine du groupe Aerospatiale se voit confier une partie homogène des éléments attribués à l'avionneur français par le GIE. Une double spécialisation produits/compétences est élaborée, évitant de la sorte la duplication des moyens industriels, améliorant le taux d'utilisation des machines et des compétences spécifiques, et diminuant les déplacements entre les usines (Kechidi, 2005). Par la suite, ce mouvement de spécialisation s'est encore accentué (Zuliani et Jalabert, 2005). Des équipes intégrées Airbus sont désignées comme responsables de fonctions globales entières (voilures, tronçon, commandes de bord, mâts réacteurs, pointe avant, etc.), alors qu'auparavant diverses équipes dispersées en Europe pouvaient avoir une part de responsabilité dans la fonction. Toutefois, cette spécialisation n'est pas parfaitement transversale aux programmes, indiquant que le processus n'est qu'entamé, comme le montre la duplication des sites d'assemblage des Airbus. Ainsi, les modèles A300/310, A320, A330/340 sont assemblés à Toulouse, les A318/319/321 à Hambourg ${ }^{16}$. En ce qui concerne 1'A380, l'assemblage est réalisé sur les chaînes françaises, la finition et l'aménagement commercial sur les chaînes allemandes, avec la duplication du centre d'essai et de mise en vol des appareils. Cette fonction d'assemblage est donc l'apanage de plusieurs sites, en contradiction avec le principe de spécialisation des sites. La persistance de cet éparpillement de la production est le produit de la volonté de

\footnotetext{
${ }^{16}$ Le plan d'économie et de restructuration « Power 8 » annoncé fin 2006 risque notamment de se traduire par le transfert à Hambourg de l'assemblage de la totalité des appareils de la famille des A320, au détriment du site toulousain. Le premier se spécialiserait ainsi dans l'assemblage des monocouloirs, le second dans les bicouloirs.
} 
chaque partie prenante, dans le respect de la logique d'arsenal, de continuer à tirer des bénéfices nationaux de cette alliance. En revanche, la conception d'ensemble de l'avion et sa commercialisation sont, quant à elles, des fonctions totalement intégrées, et ceci dans le respect de la logique de marché. Ici la logique d'arsenal, qui laisse une place aux intérêts nationaux, vient interférer avec la logique de marché visant tout à la fois à rationaliser la distribution spatiale des compétences et leur diffusion intragroupe. La recherche de la satisfaction des intérêts nationaux fait alors l'objet d'un compromis (entre Français et Allemands dans notre illustration) d'ordre politique, au détriment de la performance économique.

\section{Conclusion}

$\mathrm{Au}$ final, et sur un plan empirique, le désengagement progressif de l'Etat, les coopérations multipartites européennes engagées dès les années soixante et la spécialisation des sites qui en découlent, ouvrent la voie à la consolidation de l'industrie aéronautique européenne. L'existence du groupe EADS intégrant les industries allemande, espagnole et française en est le résultat. Et si EADS est l'aboutissement organisationnel concret de la logique de marché, il n'en reste pas moins que la proximité organisationnelle qui lie les différentes unités d'EADS est aussi le résultat de la persistance de certaines habitudes de comportements (structure bicéphale, reporting croisé) qui relèvent d'une logique d'arsenal. La structure organisationnelle est bien le reflet d'une proximité institutionnelle composée des deux logiques, l'une dominant l'autre. Les difficultés de coordination que vient de connaître EADS résultent pour partie de cette combinaison, de cette transition inachevée, induisant un défaut de coordination politique.

Sur un plan théorique cette fois, l'analyse en termes de proximités du cas EADS a permis de mettre à jour la prépondérance de la proximité institutionnelle sur les deux autres formes. Si les trois formes de proximité constituent bien trois piliers de l'action collective, il n'est pour autant nécessaire de les mobiliser toutes à la fois. Ainsi, les proximités organisationnelle et géographique peuvent jouer un rôle important dans un type de coordination, organisée et/ou localisée, et occuper une place plus réduite dans un autre, non structurée et/ou a-spatiale. Dans le cas de la production d'un produit aussi complexe qu'un avion, il a été possible de compenser une faible proximité géographique entre les sites de production par une proximité organisationnelle renforcée. Il est apparu que la proximité géographique est loin d'être indispensable à la production d'un produit pourtant complexe. En revanche, celle-ci peut s'avérer temporairement utile lors des phases de conception. La proximité organisationnelle ne suffit plus à compenser la distance physique lorsque le besoin de face à face se fait trop pressant, pour innover ensemble par exemple. Cela ne signifie pas que proximité géographique et proximité organisationnelle sont parfaitement substituables; il s'agit plutôt d'affirmer qu'elles peuvent se compléter et que leur présence simultanée n'est pas une condition impérative à l'action collective. Par contre, la proximité institutionnelle apparaît comme une condition nécessaire à toute action collective organisée ou non, étant entendu qu'elle peut varier en intensité. Dans le cas d'EADS, les contradictions portées par une proximité institutionnelle composite empêchent la formation d'une proximité organisationnelle intense et efficace. La proximité institutionnelle actuelle n'est pas encore véritablement susceptible de conduire à une action collective de strict niveau européen, dépassant définitivement les cadres nationaux. 


\section{Bibliographie}

Amable B., Palombarini S., 2004, «Une approche néo-réaliste de l'économie politique », douzième rencontre internationales du GERPISA, Comment penser la variété du capitalisme et la diversité des modèles productifs, juin, Paris.

Beckouche P., 1996, La nouvelle géographie de l'industrie aéronautique européenne, L'Harmattan, Paris.

Bazzoli L., Kirat T., 2003, «A propos du réalisme en économie des institutions et ses implications sur l'analyse des fondements juridiques des transactions économiques: Commons versus Williamson », Economie Appliquée, tome LVI, n³, pp. 171-209.

Bazzoli L., Dutraive V., 2002, «L'entreprise comme organisation et comme institution. Un regard à partir de l'institutionnalisme de J. R. Commons », Economie et Institutions, $\mathrm{n}^{\circ} 1,2^{\mathrm{e}}$ semestre, pp. 4-46.

Bessy C., 2002, Représentation, convention et institution, Centre d'Etudes de l'Emploi, Document de travail $\mathrm{n}^{\circ} 20$.

Beteille R., 1995, «Le développement de l'avion, stimulation et coopération européenne », dans E. Chadeau (éds), AIRBUS, un succès industriel européen, Institut d'Histoire de l'Industrie et Éditions Rive Droite, Paris.

Boschma R., 2005, «Does geographical proximity favour innovation?», Economie et Institutions, $\mathrm{n}^{\circ} 6$ et $7,1^{\mathrm{er}}$ et $2^{\mathrm{e}}$ semestres, pp. 111-128.

Caron A., Torre A., 2005, Réflexions sur les dimensions négatives de la proximité. Economie et Institutions, $\mathrm{n}^{\circ} 6$ et $7,1^{\mathrm{er}}$ et $2^{\mathrm{e}}$ semestres, pp. 183-220.

Cohendet P., Llerena P., Sorge A., 1992, «Technological diversity and coherence in Europe: an analytical overview », Revue d'Economie Industrielle, n59, pp. 9-26.

Commons J. R., 1934, Institutional Economics. Its Place in Political Economy, The MacMillan Compagny, New York.

Coris M., Lung Y., 2005, « Les communautés virtuelles : la coordination sans proximité ? Les fondements de la coopération au sein des communautés du logiciel », Revue d'Economie Régionale et Urbaine, $\mathrm{n}^{\circ}$ 3, pp. 397-420.

Descombes V., 1996, Les institutions du sens, Les éditions de minuit, Paris.

Dupuy C., Burmeister A., (éds), 2003, Entreprises et territoires, les nouveaux enjeux de la proximité, La Documentation Française, Paris.

Jeanneaux P., Kirat T., 2005, «Proximité, droit et conflits d'usage. Que nous apprend le contentieux judiciaire et administratif sur les dynamiques territoriales ? », Economie et Institutions, $\mathrm{n}^{\circ} 6$ et $7,1^{\text {er }}$ et $2^{\mathrm{e}}$ semestres, pp. 221-248.

Frigant V., Kechidi M., Talbot D., 2006, Les territoires de l'aéronautique, collection « Géographies en liberté », L'Harmattan, Paris.

Gilly J.P., Lung Y., 2004, «Proximités, secteurs, territoires », Quatrième Journées de la Proximité Proximité, réseaux et coordination, Marseille, juin.

Gilly J.P., Torre A., (éds.), 2000, Dynamique de proximité, L'Harmattan, Paris.

Hickie D., 2006, « Knowledge and competitiveness in the aerospace industry: the cases of Toulouse, Seattle and North-west England », European Planning Studies, vol. 14, n5, pp. 697-716. 
Kechidi M., 2005, «Mutation des relations verticales dans l'industrie aérospatiale. Une analyse de la sous-traitance d'Airbus en Midi-Pyrénées », XLIème Colloque de l'ASRDLF, septembre 2005, Dijon.

Kirat T., Lung Y, 1995, «Innovation et proximités : le territoire, lieu de déploiement des processus d'apprentissage ", dans N. Lazaric et J.M. Monnier (éds), Coordination économique et apprentissage des firmes, Paris, Economica.

Laganier R., Villalba B. et Zuindeau Z., 2002, « Le développement durable face au territoire : éléments pour une recherche pluridisciplinaire », Revue Développement Durable et Territoires, dossier $n^{\circ} 1$ Approches territoriales du Développement Durable.

Leriche F., Zuliani J.M., 2007, «L'industrie aéronautique toulousaine et la déplétion pétrolière : quelles perspectives ? », Géographie, Economie, Société, n9, pp. 19-38.

Mitchell W., 1935, «Commons on Institutional Economics», American Economic Review, vol. 25, December, pp. 635-652.

Muller P., 1988, Airbus l'ambition européenne, logique d'Etat, logique de marché, L'Harmattan, Paris.

Palloix C., 2000, "Les ouvertures de l'institutionnalisme : apports et limites », colloque Institutions et Organisations, ERSI-CRIISEA (Université Picardie) et GERME (Université Paris 7), mai, Amiens.

Pecqueur B., 1997, «Processus cognitifs et construction des territoires économiques », dans B. Guilhon, P. Huard, M. Orillard et J. B. Zimmermann (éds.), Economie de la connaissance et des organisations, Paris, L'Harmattan.

Pecqueur B., Zimmermann J. B., 2004, «Introduction. Les fondements d'une économie de proximités ", dans B. Pecqueur et J. B. Zimmermann (éds.), Economie de Proximités, Hermès, Lavoisier, Paris.

Rallet A., 1999, «L'économie de proximités », communication à l'école-chercheur INRA, Le Croisic, 8-10 décembre.

Rallet A., Torre A., 2007, « Introduction. Faut-il être proche pour innover ensemble ? », dans A. Rallet et A. Torre (éds), Quelles proximités pour innover ?, L’Harmattan, Paris.

Reynaud B., 2001, "Suivre des règles dans les organisations », Revue d'Economie Industrielle, $4^{\mathrm{e}}$ trimestre, pp. 53-68.

Searle J., 1998, La construction de la réalité sociale, traduit de l'anglais par C. Tiercelin, Gallimard, Paris.

Théret B., 2001, «Saisir les faits économiques : la méthode Commons », dans Lecture de John R. Commons, Cahiers d'économie politique, n40-41, L’Harmattan, Paris.

Talbot D., 2005, « Les proximités, entre contrainte et libération de l'action : le cas d'EADS », Economie et Institutions, $\mathrm{n}^{\circ} 6$ et 7, pp. 153-182.

Talbot D., Kirat T., (éds), 2005, «Proximités et Institutions : nouveaux éclairages », Economie et Institutions, $\mathrm{n}^{\circ} 6$ et 7 .

Torre A., Rallet A., 2005, «Proximity and localization », Regional Studies, vol. 39, n 1, pp. 47-60.

Zuliani J.M., Jalabert G., 2005, «L’industrie aéronautique européenne : organisation industrielle et fonctionnement en réseaux », L’Espace géographique, n² 2 , pp. 117-133. 
Carte 1 Les principaux sites de production d'Airbus SAS en 2006

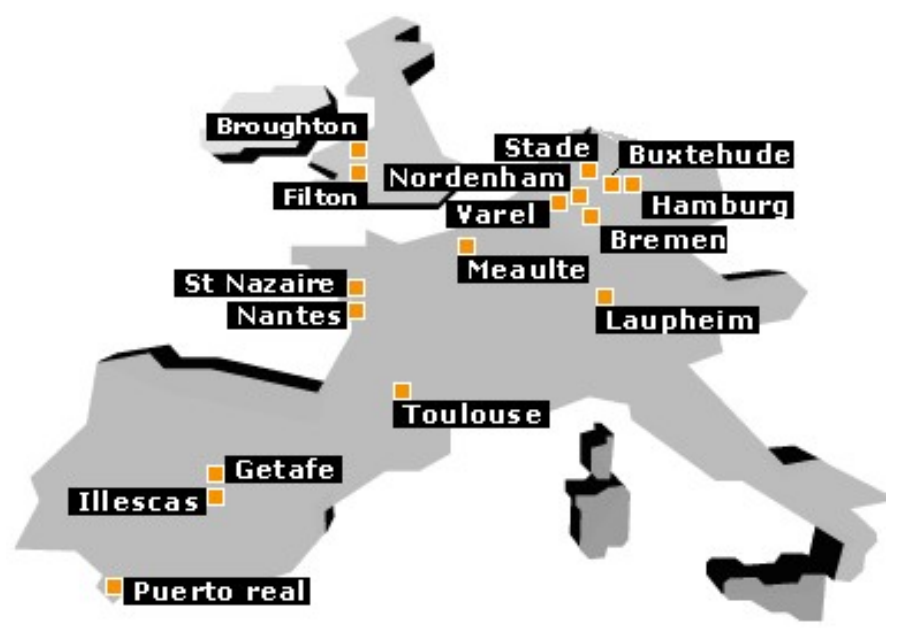

Source : www.airbus.com 
Figure 1 Le transport (simplifié) des éléments de l'A380 par voies maritime, aérienne et terrestre

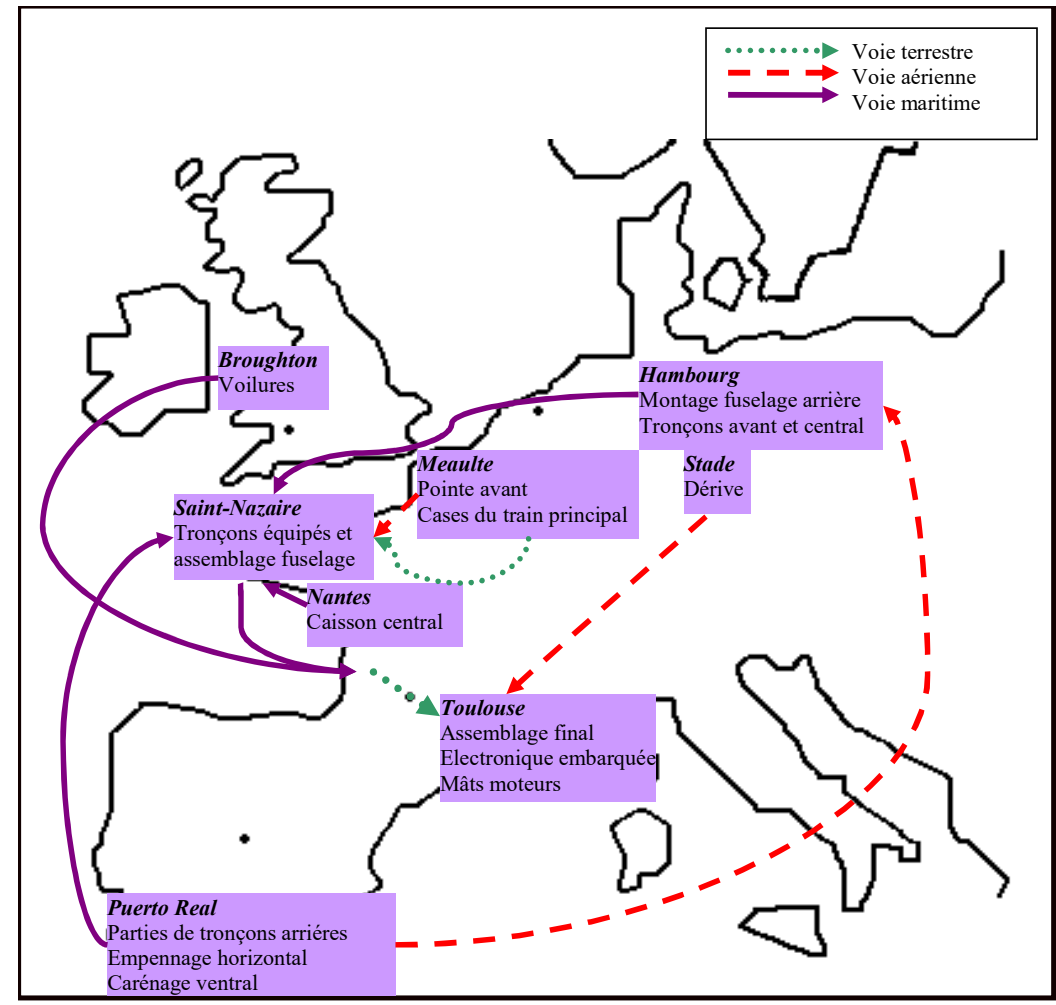

Source : auteur. 